\title{
Growth of Arabidopsis flavonoid mutants under solar radiation and UV filters ${ }^{25}$
}

\author{
Edwin L. Fiscus a,b,*, Robert Philbeck a, Anne B. Britt ${ }^{c}$, \\ Fitzgerald L. Booker ${ }^{\mathrm{a}, \mathrm{b}}$ \\ a United States Department of Agriculture, Agricultural Research Service, 3908 Inwood Road, Raleigh, NC 27603, USA \\ ${ }^{\mathrm{b}}$ Crop Science Department, North Carolina State University, Raleigh, NC 27695-7620, USA \\ ${ }^{\mathrm{c}}$ Section of Plant Biology, University of California, Davis, Davis, CA 95616, USA
}

Received 15 September 1998; received in revised form 1 February 1999; accepted 8 February 1999

\begin{abstract}
Growth of the chalcone isomerase defective tt-5 mutant of Arabidopsis thaliana and its Landsberg erecta progenitor were compared under a variety of full spectrum solar radiation conditions to determine if the tt-5 mutant could serve as an adequate subject for studies of the mechanisms of damage by UV-B radiation. An experiment was conducted in the fall of 1995 under open field filter frames using cellulose diacetate and Mylar filters to transmit and exclude natural UV-B irradiation, respectively. Even though growth under these conditions was slow and erratic owing to lack of temperature control, growth suppression as indicated by rosette diameter and harvest fresh weights provided a sensitive indicator of UV-B stress. This experience led to development of temperature-controlled Teflon-covered field chambers that admit up to $88 \%$ of the total daily PAR and about $85 \%$ of ambient UV-B, omit predators, and provide a generally stable environment for quantitative plant growth studies. The chambers were designed to facilitate the addition of optical filters and/or shade cloth and to accommodate control of the gaseous environment for pollutant and climate change studies and to provide clean air for other experiments. Three additional experiments were conducted in these chambers. Measurements of rosette diameter, weights of various aboveground plant parts, and plant height were evaluated as potential methods of comparing growth sensitivities of the tt-5 mutant to UV-B radiation. The weight of the reproductive parts (flowers and siliques) as a fraction of the total (e.g. harvest index) was consistently and negatively affected by solar UV-B, as was simple plant height. However, in no case, even in the virtual absence of UV-B, was growth of tt-5 comparable to that of Ler. We conclude that the disruption of secondary metabolism in tt-5 has growth implications far beyond the lack of UV-B protection, making it unsuitable as a surrogate for high UV-B experimentation. (C) 1999 Elsevier Science B.V. All rights reserved.
\end{abstract}

Keywords: Arabidopsis; Flavonoid mutants; Radiation; Spectral balance; UV-B

\footnotetext{
Mention of a product or company name does not constitute an endorsement by the United States Government, North Carolina State University or the University of California at Davis nor imply any criticism of other products not mentioned.

* Corresponding author. Present address: United States Department of Agriculture, Agricultural Research Service, 3908 Inwood Road, Raleigh, NC 27603, USA. Tel.: + 1-919-515-3505; fax: + 1-919-460-7311.

E-mail address: edfiscus01@sprymet.com (E.L. Fiscus)
} 


\section{Introduction}

The small size of Arabidopsis thaliana (L.) Heynh, short generation time and small genome have made it a popular model system for the genetic analysis of plant responses to abiotic stresses. Arabidopsis mutants with defects in growth responses to light or to cold, in the detoxification of reactive oxygen species, in the absorption of UV-B radiation, and in the repair of UV-induced DNA damage have all provided insights into the molecular mechanisms of stress resistance. Although the biological significance of these stress resistance or developmental responses, in terms of their effect on overall plant growth, can be determined quantitatively in the laboratory, it is sometimes difficult to extrapolate from results obtained in the laboratory to expected results in the field. Demonstrating the usefulness of genetically isolated physiological characteristics or control mechanisms in a more natural setting should enhance the efficiency with which we can distinguish between environmental responses that are recognizable only in an artificial, optimized laboratory environment, and those that are critical to plant growth and yield in the field.

Because of current interest in the mechanisms of damage by UV-B radiation and the difficulties of setting up realistic exposure systems, we thought that the chalcone isomerase defective transparent testa mutant tt-5 (Koornneef, 1981) might serve as a surrogate for high UV-B treatments since it has a much reduced capacity to synthesize flavonoids and sinapic acid esters which are also thought to be important UV-absorbing compounds (Shirley et al., 1992; Li et al., 1993; Landry et al., 1995). These compounds which tend to be localized in the epidermis can filter out $90 \%$ or more of the ambient UV-B radiation (Robberecht and Caldwell, 1978) thus protecting the leaf mesophyll from serious damage. In their absence, a given level of UV-B exposure should have a much greater physiological effect in tt-5 than in the wild ecotype (Landsberg erecta (Ler)) which does synthesize these compounds. In fact, all other factors being equal, normal levels of solar radiation could be used to produce UV-B mesophyll tissue exposures in tt-5 plants of roughly ten times that possible in the wild type, without the use of supplemental exposure systems. Indeed, laboratory experiments have demonstrated this type of UV-B hypersensitivity. In the experiments of $\mathrm{Li}$ et al. (1993) growth suppression of tt-5 relative to Ler was demonstrated at relatively low UV-B levels. However, they also observed growth suppression of about $18 \%$, averaged over three experiments, in the complete absence of UV-B. Thus, even though they did demonstrate hypersensitivity to UV-B radiation, the growth of tt-5 was not comparable to Ler in the absence of UV-B and raises the question of its suitability for use in experiments designed to discover mechanisms by which plants respond to $\mathrm{UV}-\mathrm{B}$ radiation.

Another troubling feature of laboratory experiments designed to discover UV-B response mechanisms is the difficulty in achieving a realistic balance between UV-A and the visible parts of the solar spectrum during an experiment. The importance of doing so is clear from numerous reports that UV-B induced damage, which is clearly manifest in growth chambers and greenhouses can be moderated or eliminated when plants are grown under PAR levels approaching outdoor conditions (reviewed by Fiscus and Booker, 1995). Caldwell et al. (1994) not only demonstrated a threshold level for PAR, below which UV-B damage was apparent, but that UV-A could substitute for PAR in this protective capacity. It has also been demonstrated that adequate levels of UV-A and/or PAR, as well as specific red, blue or green wave bands, can stimulate flavonoid production (reviewed by Fiscus and Booker, 1995). Thus the spectral balance as well as the overall intensity of the exposure system become important considerations if we are interested in studying 'normal' radiation responses. Also, since tt-5 is defective in chalcone isomerase, a substantial part of secondary metabolism is blocked, in addition to synthesis of UV-absorbing compounds, and there is little information on how that might affect growth. Therefore, a series of experiments were conducted to determine if the tt-5 mutant would serve as suitable material for studying UV-B responses and damage mechanisms with a high degree of confidence that there are no significant 
confounding physiological differences other than the lack of UV-B absorbing compounds to account for the responses. In order to test this criterion of suitability, we hypothesized that at adequate levels of visible light and in the absence of UV-B the tt-5 mutant would grow as well as Ler.

\section{Materials and methods}

Simply growing Arabidopsis out of doors poses several special problems for the investigator, including insect predation and excessively high (often lethal) temperatures. In this paper, we relate the growth of Arabidopsis in the field under filters which either transmit or exclude UV-B, then describe the characteristics and use of small Tefloncovered, temperature-controlled field chambers used for growing Arabidopsis. The Teflon covering transmits most of the UV and visible solar radiation spectrum allowing plants to grow under a natural balance of solar radiation when no exclusion filters are in place. The purpose of the chambers is to provide facilities for the realistic testing of UV-B sensitives and other mutants with sensitivities or resistances to environmental parameters such as air pollutants, elevated $\mathrm{CO}_{2}$, temperature extremes, and/or combinations. For that purpose, the air handling system has been fitted with charcoal filters to accommodate studies of gaseous pollutants and to provide a pollutionfree environment for all other experiments. Since each system is small, it also should be easy to control $\mathrm{CO}_{2}$ levels, above and below ambient, for climate change studies. These chambers differ from those described by Ryan and Ireland (1997) in that theirs had no temperature control or capacity for modification of the gaseous environment. However, their chambers did have a supplemental UV-B system using an important and useful new modulation technique.

\subsection{Field radiation filters}

Our early attempts to grow UV-sensitive mutants in the field were conducted under filters constructed of ordinary $90 \times 150$-cm aluminum window screens which provided about $50 \%$ shade for both visible and UV radiation. The long axis of the frames was oriented east to west and the frame supported by posts at the corners. The south edge was anchored about $15 \mathrm{~cm}$ above grade and the whole frame inclined at an angle of $30^{\circ}$ with the high edge to the north. Either Mylar (0.13 mm Polyester film; Cadillac Plastic and Chemical, Baltimore, MD) or cellulose diacetate (0.13 mm; Cadillac Plastic and Chemical) (CD) sheets were then clipped to the screen and allowed to hang over the ends to minimize UV-B incursion early and late in the day. The north and south edges were left uncovered, allowing air circulation beneath the filters. When placed beneath the Mylar filter, plants received no direct UV-B radiation but it was not possible to exclude diffuse radiation and still allow air circulation. Ambient PAR was continuously monitored using a LICOR quantum sensor (LI-COR, Lincoln, NE) while ambient UV was monitored with a UV-B Pyranometer (Model UVB-1, Yankee Environmental Systems, Montague, MA). Both units were interfaced to a data acquisition system that stored average environmental data at 2-min intervals. Because it was not possible to monitor radiation beneath the filters during this experiment, the following procedure was used to estimate the UV-B and PAR exposures. Spot comparisons of ambient UV-B to UV-B radiation beneath the filters were made three times during the experiment using portable UV-B sensors incorporating solar blind photo tubes (model R1228, Hamamatsu, Middlesex, NJ). The response from each individual sensor was calibrated against the UV-B Pyranometer. Then, using the ratio of ambient:filter UV-B determined by the spot checks, UV-B radiation beneath the filters was estimated throughout the experiment from the ambient UV$B$ pyranometer readings. PAR levels beneath the filters were estimated in the same way except that a portable LI-COR quantum sensor and readout were used to establish the ambient:filter ratio.

\subsection{Growth chambers}

Each chamber (Fig. 1) was built on a concrete pad in the field and consisted of a $1.22 \times 2.44-\mathrm{m}$ 
growth area covered with a rounded lid hinged along one side to allow opening. The lid frames were constructed of welded $5.08 \times 2.54 \times 0.32-\mathrm{cm}$ aluminum channel. The roof bows were $1.27-\mathrm{cm}$ EMT conduits stabilized at the peaks with a $1.90-\mathrm{cm}$ aluminum channel ridge running the length of the chamber.

The lid was covered with a single layer of 0.0127-cm (5-mil) thick FEP Teflon film (E.I. duPont de Nemours, Wilmington, DE) secured with aluminum Kwic-Klip Film Fastener (Jāderloon, Irmo, SC) bases and caps which allow additional coverings such as shade cloth and/or filters to be affixed. The lid could be secured in the open position with a support rod at each end of the chamber. Each support was attached to a pivot point on the lid frame and another on the chamber base. The center of the rod was jointed and could be held in the extended position with a sliding steel collar. The $30.5-\mathrm{cm}$ high base walls were constructed of $3.81-\mathrm{cm}$ thick styrofoam with aluminum skin on both sides (APT-O-LITE Insulated Panels, APT Industries, Charlotte, NC). The base was attached to the concrete slab, trimmed with 5.08-cm aluminum angle and caulked to eliminate incursion of air, water and plant preda-

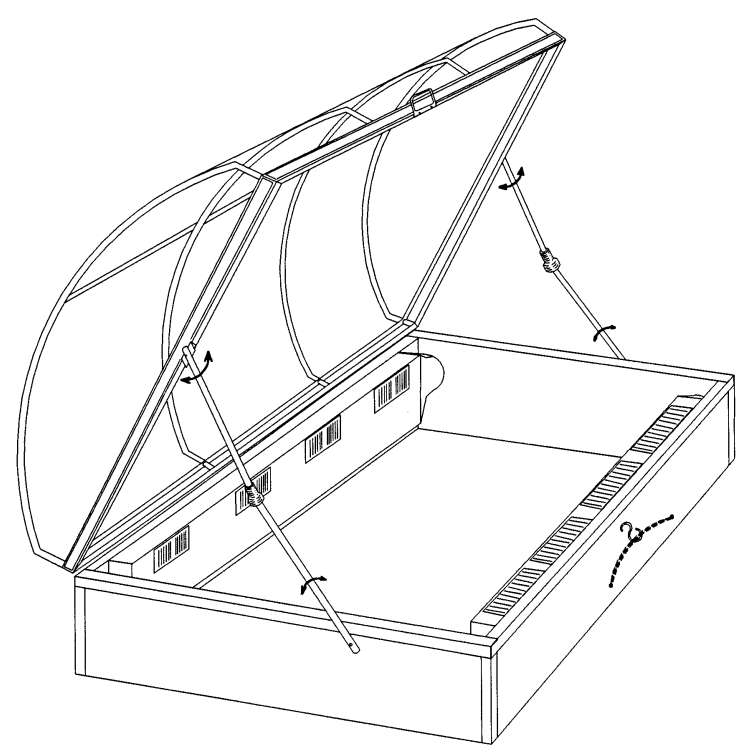

Fig. 1. The Teflon-covered outdoor growth chamber. Air handling and conditioning units are exterior. tors. The contact area between the base and the lid was also lined with closed cell foam for the same reasons.

The growth region of the chambers was connected to the external heating and cooling units through 15.24-cm circular galvanized ducts projecting through the north wall of the chambers. The air supply and return ducts were $8.26 \times 25$.4$\mathrm{cm}$ galvanized wall stacks running the length of the chambers so that air flow was across the chamber rather than lengthwise. The supply side duct had four directionally adjustable inlet vents while the four return vents were not adjustable.

Temperature control in chamber experiments 1 and 2 was provided by a $4.1-\mathrm{kW}$ cooling unit coupled with a $1.55-\mathrm{kW}$ finned heater. The cooling units were upgraded to $5.3 \mathrm{~kW}$ for experiment 3. Air flow was driven by dual centrifugal blowers with a no-load rating of $0.26 \mathrm{~m}^{3} \mathrm{~s}^{-1}$. All the external heating, ventilation and air-conditioning components and duct-work were insulated.

Chamber air temperatures were monitored with thermocouples placed in the air-return ducts. In experiment 1 , PAR and UV-B radiation were monitored continuously outside the chambers and inside, under both UV-B filter treatments. In experiments 2 and 3, PAR and UV-B were monitored both outside and inside the chambers. However, in these experiments, only one pair of sensors was used inside each chamber since each chamber was completely covered with the appropriate filter material and there were no subplots within the chambers, except for genotype. PAR was measured with quantum sensors (LI-COR), all of which had been secondarily calibrated against a factory calibrated unit and cross checked against a spectroradiometer (model 742, Optronics Laboratories, Orlando, FL). UV-B was monitored with the solar blind photo tubes previously mentioned. The response from each individual UV-B sensor was calibrated against the UV-B Pyranometer (Model UVB-1, Yankee Environmental Systems). Using the spectral response of the UV-B pyranometer, readings were converted ratiometrically to other biologically relevant weighted irradiances such as Caldwell's generalized plant action spectrum normalized to $300 \mathrm{~nm}$ (Caldwell, 1971) (PAS300) or the alfalfa DNA 
damage spectrum (QDNA) of Quaite and Sutherland (1992). Action spectrum ratios were calculated using the UV radiative transfer model 'UV-B' (Fiscus and Booker, 1994) based on the algorithms of Green (1983) and the original code of Björn and Murphy (1985). Thus, both the UV-B pyranometer readings and photo-diode sensor readings were converted to PAS300 and QDNA irradiances.

\subsection{Plant culture and measurements}

\subsubsection{General plant culture}

Plants used in the field filter experiment were cultured in the same way as the three chamber experiments which were conducted with slight variations between them. The Arabidopsis seeds were sown on the surface of $10-\mathrm{cm}$ diameter pots filled with a commercial potting mixture (Sunshine Mix \#1, SunGro Horticulture, Bellevue, WA) which had previously been saturated with a commercial liquid fertilizer (Miracle-Gro, Stern's Miracle-Gro Products, Port Washington, NY) and allowed to drain. Plants treated under the field-filters were cultured in the same way except that the pots were placed in flats under $70 \%$ shade cloth and later transferred to the cellulose diacetate and Mylar filter frames. In the chamber experiments, pots were placed in the Teflon-covered chambers in flats supported at the height of the top of the chamber side walls so there was no obstruction of ambient radiation by the walls. In all experiments, after plants had reached the fourleaf stage they were thinned so that for the remainder of the experiment there was only one plant per pot. When necessary throughout the experiment the pots were subirrigated in the flats. At about 25 days after planting (DAP) the plants were also fertilized by subirrigating with MiracleGro plant food solution.

\subsubsection{Field filter experiment}

Seeds were sown on 8 October 1995. During germination and early growth there was no differential exclusion of UV-B radiation, although the plants were under $70 \%$ shade. Starting at 41 DAP and continuing until final harvest, the maximum point-to-point distance of each basal rosette was measured about three times per week. In the rest of this paper, these measurements are referred to as the rosette diameter and were thought to provide a good non-destructive indicator of plant growth and response to environmental stressors. Plants were maintained under the $70 \%$ shade treatment until after the diameter measurements at 53 DAP when they were transferred to the filter frame assemblies. Half of the plants of each genotype were placed under the Mylar filters and half under $C D$, where they remained until harvest. After diameter measurements at 95 DAP the rosettes were separated from the roots and the rosette fresh weight measured.

\subsubsection{Chamber experiment 1}

Seeds were sown on 12 May 1997. Two separate chambers were used, one was covered with a $30 \%$ shade cloth while the second was not shaded. In each chamber the plant flats were segregated into two groups. During germination and early growth each group, in both chambers, was shielded from direct UV-B radiation by sheets of Mylar which were supported inside the chambers approximately $20 \mathrm{~cm}$ above the soil surface. Rosette diameter measurements started at 23 DAP and continued until final harvest. Once the plants had become established and were growing rapidly (28 DAP), the Mylar was removed from one group in each chamber thus exposing them to direct UV-B radiation. Final harvest was performed at 40 DAP by separating the shoot from the root just below the basal rosette. The shoot was then separated into: the rosette; leaves other than the rosette; flowering stalks; and reproductive parts (flowers and siliques). Fresh weights were then measured for each group of parts. Roots were discarded.

\subsubsection{Chamber experiment 2}

Seeds were sown on 7 August 1997. Two chambers were used, both covered with $30 \%$ shade cloth. During germination and early growth, all transparent chamber surfaces were covered with exterior-mounted Mylar sheets sandwiched between the shade cloth and the Teflon covers. Rosette diameter measurements were made on alternate days starting at 18 DAP and continuing 
until harvest. Late on 26 DAP the Mylar sheets on one chamber were replaced with $\mathrm{CD}$, exposing the plants in that chamber to direct UV-B radiation. In this situation, the acetate had no relevant selective filtration effects but did act as a control for the general attenuation of the Mylar across the UV-A and visible parts of the spectrum. At harvest (39 DAP) final plant height was measured and the shoots were again cut from the roots but only separated into reproductive and 'other' parts. The former included flowers and siliques while the latter included rosettes, stalks and other leaves. Roots were left in the soil and discarded. After determining the fresh weights, the parts were pooled, dried in an oven at $60^{\circ} \mathrm{C}$ until constant weight and the total dry weight determined.

\subsubsection{Chamber experiment 3}

Seeds were sown on 11 December 1997. Two chambers were used, both covered with a $30 \%$ shade cloth. One chamber was covered with a Mylar filter while the other was covered with a transparent polyvinyl chloride filter $(0.20-\mathrm{mm}$ Krene PVC film with UV inhibitors; Livingston Coating, Charlotte, NC) (PVC) which takes out UV-B and UV-A to about $340 \mathrm{~nm}$. Rosette diameter measurements were begun at 25 DAP and made three times per week until harvest. After the rosette measurements at 40 DAP all the plants were swapped between chambers so that the ones previously growing under Mylar were placed in the PVC chamber and vice versa. Rosette measurements were continued until 65 DAP. After the final rosette diameter measurements the plants were discarded.

\subsection{Biochemical analyses}

As a quick check on the reported differences between genotypes and on UV-B treatment effects, we examined the absorbance of tissue extracts at $300 \mathrm{~nm}$ as well as the total phenolic compounds present in the tissues of the $30 \%$ shade treatment of experiment 1 . Absorbance at $300 \mathrm{~nm}$ should provide an index for the quantity of UV-B protective compounds in the tissue since it is in the middle of the UV-B band and has a biologically effective value of 1 according to Cald- well's generalized plant action spectrum (Caldwell, 1971). Phenolic content was examined because the total is comprised mainly of flavonoids, phenolic acids and hydroxycinnamic acid esters which absorb strongly in the UV-B band.

Soluble components were extracted from tissue samples which were freeze-dried and ground to pass a $0.5-\mathrm{mm}$ mesh screen. Samples $(50 \mathrm{mg})$ were extracted three times with $1 \mathrm{ml}$ of $50 \%$ aqueous methanol, with mixing for $5 \mathrm{~min}$ at $25^{\circ} \mathrm{C}$ each time. Following each extraction, the insoluble material was pelleted by centrifugation $(16000 \times g, 5$ $\mathrm{min}$ ), and the supernatants were pooled by sample. Samples were then filtered through $0.45-\mu \mathrm{m}$ nylon filters and immediately assayed.

Total phenolic concentration in the soluble fraction was determined by the Folin-Ciocalteu method (Singleton and Rossi, 1965). An aliquot $(50 \mu \mathrm{l})$ of each sample was mixed with $0.475 \mathrm{ml}$ of $0.25 \mathrm{~N}$ Folin-Ciocalteu reagent (Sigma) followed 3 min later by $0.475 \mathrm{ml}$ of $1 \mathrm{M} \mathrm{Na}_{2} \mathrm{CO}_{3}$. After 1 $\mathrm{h}$, absorbance of the solutions at $724 \mathrm{~nm}$ was measured. 4-Coumaric acid was used to prepare a standard curve, and assay results were expressed as 4-coumaric acid equivalents. To measure UVB-absorbing compounds, $100 \mu \mathrm{l}$ of each sample was diluted to $1 \mathrm{ml}$ with $50 \%$ methanol and absorbance at $300 \mathrm{~nm}$ was measured.

\subsection{Experimental design and statistical analysis}

The field filter experiment and chamber experiments 2 and 3 were designed as split plots with the UV filters (Mylar and Cellulose diacetate in the field filter experiment and experiment 2; Mylar and PVC in experiment 3) as the main plot and genotype (Ler and tt-5) as the sub plot. Genotypes within the sub plot were randomly distributed. Plant data from each experiment were analyzed separately using a two-way analysis of variance followed by Tukey's all-pairwise comparison. Chamber experiment 1 was designed as a split-split plot with shade (0 and $30 \%)$ as the main plot, UV-B filters as the sub plot and genotype as the sub-sub plot. In this experiment the chambers were separated by filter treatments into their north and south ends, so the arbitrary choice 
Table 1

Mean daily radiation levels under the open field-filter frames ${ }^{\mathrm{a}}$

\begin{tabular}{|c|c|c|c|}
\hline Treatment & $\operatorname{PAR}\left(\mathrm{mol} \mathrm{m}{ }^{-2} \mathrm{day}^{-1}\right)$ & QDNA $\left(\mathrm{kJ} \mathrm{m}^{-2} \mathrm{day}^{-1}\right)$ & $\operatorname{PAS} 300\left(\mathrm{~kJ} \mathrm{~m}^{-2} \mathrm{day}^{-1}\right)$ \\
\hline \multicolumn{4}{|c|}{ Period $1(0-40 D A P)$} \\
\hline $70 \%$ Shade & $6.94(0.49)$ & $0.489(0.044)$ & $0.238(0.066)$ \\
\hline \multicolumn{4}{|c|}{ Period 2 (41-95 DAP) } \\
\hline Ambient & $17.28(2.04)$ & $1.280(0.090)$ & $0.512(0.036)$ \\
\hline Mylar & $7.88(0.66)$ & $0.138(0.010)$ & $0.055(0.004)$ \\
\hline
\end{tabular}

${ }^{a}$ Each number is the mean of the daily integrals over the specified periods followed by the standard error in parentheses. Only ambient levels were monitored and other values estimated from UV-B and PAR measurements made under the filters three times during the experiment. Biologically effective UV radiation is expressed both as the Quaite and Sutherland (1992) DNA damaging radiation and Caldwell's plant action spectrum normalized to $300 \mathrm{~nm}$.

was made for the Mylar treatment to be in the north end of one chamber and the south end of the other. Genotypes were randomized within each filter sub plot. Plant data were analyzed using a two-way analysis of variance followed by Tukey's all-pairwise comparison. The number of replicates varied among experiments from 7 to 19 and are given in the plant data tables in Section 3. All data were tested for homogeneity of variance and normality prior to analysis. Statistical tests were considered significant if $P \leq 0.05$.

\section{Results}

\subsection{Field filter performance}

Estimated daily average radiation conditions for the field filter experiment are shown in Table 1. Under the shade cloth used for the initial growth period (period 1), both PAR and UV-B were attenuated to about the same extent $(69 \%)$. Both the CD and Mylar in the filter-screen frames attenuated ambient PAR by about $55 \%$, but UV$\mathrm{B}$ was attenuated by $62 \%$ under CD compared to $89 \%$ under the Mylar.

\subsection{Chamber performance}

Average PAR and UV-B radiation transmission characteristics of the chamber covers is illustrated in Table 2 and for a moderately clear day in Fig.
2. During experiment 1 , daily PAR transmission (Fig. 2A and Table 2) was, on average, about $88 \%$ of the total daily ambient levels. Of the $12 \%$ attenuation, about $40 \%$ was due to the Teflon covering and the rest to structural supports and possibly to some differential reflection from the Teflon. The prominent shadow about midday was caused by the main north-south cross-member and since it was impossible to form a perfectly smooth cover with no sags or wrinkles, incoming and reflected outgoing radiation could easily experience highly variable angles of incidence resulting in some differential reflection from the Teflon. The latter factor would also account for the higher noise levels of the measurements inside the chambers (Fig. 2A). The presence of the Mylar UV-B shields also attenuated PAR another 14\% so that the PAR levels reaching the soil surface beneath the Mylar were $74 \%$ of ambient. The $30 \%$ shade cloth covering chamber \#1 further reduced PAR in that chamber to $54 \%$ of ambient under the Mylar and to $62 \%$ without Mylar. Similar figures were determined for experiment 2 except that: (1) covering the entire chamber with Mylar was much more effective as a UV-B screen than trying to subdivide each chamber; and (2) swapping the Mylar for the CD compensates very well for the UV-A and visible radiation attenuation of the Mylar but still exacts a penalty in UV-B transmission, thus limiting the severity of the treatment. Covering a chamber with a PVC filter reduces PAR to about the same extent as 
Mylar but attenuates the UV-B to the noise level of the sensors. Further details of the chamber radiation characteristics are given in Table 2.

Chamber air temperature control was generally satisfactory. Although thermostats were set for a nominal $20^{\circ} \mathrm{C}$ there was control cycling around the set-point with a range of less than $\pm 2{ }^{\circ} \mathrm{C}$. On an extreme day when the air temperature reached about $38^{\circ} \mathrm{C}$ with a high radiation load the cooling capacity of the system was still capable of holding the chamber to a maximum temperature of $<$ $24^{\circ} \mathrm{C}$, a temperature that, if not ideal for Arabidopsis, still protected the plants from high temperature stress and allowed the experiment to proceed. Of course, warmer climates will require greater cooling capacity, especially if skies tend to be clear during the warmest part of the year. Actual chamber daily temperature means and standard deviations over the period of experiment 1 averaged $19.9 \pm 1.45$ and $21.2 \pm 1.26^{\circ} \mathrm{C}$ for the shaded and unshaded chamber, respectively. For experiment 2 with both of the chambers shaded, the average temperatures for chambers 1 and 2 were $19.9 \pm 1.28$ and $20.9 \pm 1.24^{\circ} \mathrm{C}$, respectively. With the larger cooling units and improved control systems used in experiment 3 and subsequent experiments, the temperature range was generally less than $\pm 0.5^{\circ} \mathrm{C}$ and chamber temperatures re-

Table 2

Mean daily radiation levels in the chambers and surroundings during chamber experiments 1,2 and $3^{\mathrm{a}}$

\begin{tabular}{|c|c|c|c|c|c|}
\hline Chamber \# & 1 & & 2 & & Ambient \\
\hline \multicolumn{6}{|l|}{ Experiment 1} \\
\hline Period $1(0-28 D A P)$ & Mylar & No Filter & Mylar & No Filter & \\
\hline PAR $\left(\mathrm{mol} \mathrm{m}^{-2} \mathrm{day}^{-1}\right)$ & $23.45(1.65)$ & & $33.38(2.27)$ & & $43.69(3.07)$ \\
\hline QDNA $\left(\mathrm{kJ} \mathrm{m}^{-2} \mathrm{day}^{-1}\right)$ & $0.91(0.07)$ & & $1.02(0.06)$ & & $5.35(0.34)$ \\
\hline PAS300 $\left(\mathrm{kJ} \mathrm{m}^{-2} \mathrm{day}^{-1}\right)$ & $0.66(0.05)$ & & $0.74(0.05)$ & & $3.88(0.24)$ \\
\hline \multicolumn{6}{|l|}{ Period 2 (29-40 DAP) } \\
\hline $\operatorname{PAR}\left(\mathrm{mol} \mathrm{m}^{-2} \mathrm{day}^{-1}\right)$ & $24.61(1.40)$ & $28.83(1.61)$ & $34.62(1.93)$ & $40.88(2.28)$ & $46.80(2.61)$ \\
\hline QDNA $\left(\mathrm{kJ} \mathrm{m}^{-2}\right.$ day $\left.^{-1}\right)$ & $0.97(0.08)$ & $3.74(0.22)$ & $1.11(0.07)$ & $5.21(0.32)$ & $5.85(0.35)$ \\
\hline PAS300 $\left(\mathrm{kJ} \mathrm{m}^{-2}\right.$ day $\left.^{-1}\right)$ & $0.74(0.06)$ & $2.91(0.18)$ & $0.85(0.06)$ & $3.99(0.26)$ & $4.48(0.28)$ \\
\hline Experiment 2 & Mylar & & Mylar & CD & \\
\hline \multicolumn{6}{|l|}{ Period $1(0-26$ DAP $)$} \\
\hline $\operatorname{PAR}\left(\mathrm{mol} \mathrm{m}^{-2} \mathrm{day}^{-1}\right)$ & $24.49(0.77)$ & & $24.22(0.76)$ & & $44.47(1.37)$ \\
\hline QDNA $\left(\mathrm{kJ} \mathrm{m}^{-2} \mathrm{day}^{-1}\right)$ & $0.27(0.02)$ & & $0.30(0.20)$ & & $5.25(0.16)$ \\
\hline PAS300 $\left(\mathrm{kJ} \mathrm{m}^{-2} \mathrm{day}^{-1}\right)$ & $0.20(0.02)$ & & $0.23(0.01)$ & & $4.01(0.12)$ \\
\hline \multicolumn{6}{|l|}{ Period 2 (27-39 DAP) } \\
\hline $\operatorname{PAR}\left(\mathrm{mol} \mathrm{m}{ }^{-2} \mathrm{day}^{-1}\right)$ & $20.39(1.52)$ & & & $21.38(1.62)$ & $37.67(2.74)$ \\
\hline QDNA $\left(\mathrm{kJ} \mathrm{m}^{-2} \mathrm{day}^{-1}\right)$ & $0.33(0.05)$ & & & $1.74(0.23)$ & $4.25(0.30)$ \\
\hline PAS300 $\left(\mathrm{kJ} \mathrm{m}^{-2} \mathrm{day}^{-1}\right)$ & $0.25(0.04)$ & & & $1.30(0.17)$ & $3.16(0.22)$ \\
\hline Experiment 3 & PVC $\rightarrow$ Mylar & & Mylar $\rightarrow$ PVC & & \\
\hline \multicolumn{6}{|l|}{ Period $1(0-40 D A P)$} \\
\hline $\operatorname{PAR}\left(\mathrm{mol} \mathrm{m}{ }^{-2} \mathrm{day}^{-1}\right)$ & $9.90(0.82)$ & & $8.92(0.71)$ & & $14.76(1.25)$ \\
\hline QDNA $\left(\mathrm{kJ} \mathrm{m}^{-2} \mathrm{day}^{-1}\right)$ & 0 & & $0.06(<0.01)$ & & $0.68(0.11)$ \\
\hline PAS300 $\left(\mathrm{kJ} \mathrm{m}^{-2} \mathrm{day}^{-1}\right)$ & 0 & & $0.06(<0.01)$ & & $0.66(0.06)$ \\
\hline \multicolumn{6}{|l|}{ Period 2 (41-65 DAP) } \\
\hline $\operatorname{PAR}\left(\mathrm{mol} \mathrm{m}^{-2} \mathrm{day}^{-1}\right)$ & $11.22(1.54)$ & & $12.85(1.77)$ & & $16.05(2.16)$ \\
\hline QDNA $\left(\mathrm{kJ} \mathrm{m}^{-2} \mathrm{day}^{-1}\right)$ & 0 & & $0.08(0.01)$ & & $0.90(0.11)$ \\
\hline PAS300 $\left(\mathrm{kJ} \mathrm{m}^{-2} \mathrm{day}^{-1}\right)$ & 0 & & $0.08(0.01)$ & & $0.89(0.11)$ \\
\hline
\end{tabular}

\footnotetext{
${ }^{\text {a }}$ In experiment 1, chamber 1 was covered with 30\% shade cloth and Mylar shields were removed from half the population in each chamber to initiate treatments (period 2). In experiment 2, both chambers were covered with $30 \%$ shade cloth and treatments were initiated by replacing the Mylar on chamber 2 with CD. In experiment 3, both chambers were covered with $30 \%$ shade cloth while chamber 1 was covered with a PVC filter and chamber 2 was covered with a Mylar filter. All plants were swapped between chambers at DAP 40. Each number is the mean of the daily integrals over the specified periods followed by the standard error in parentheses.
} 


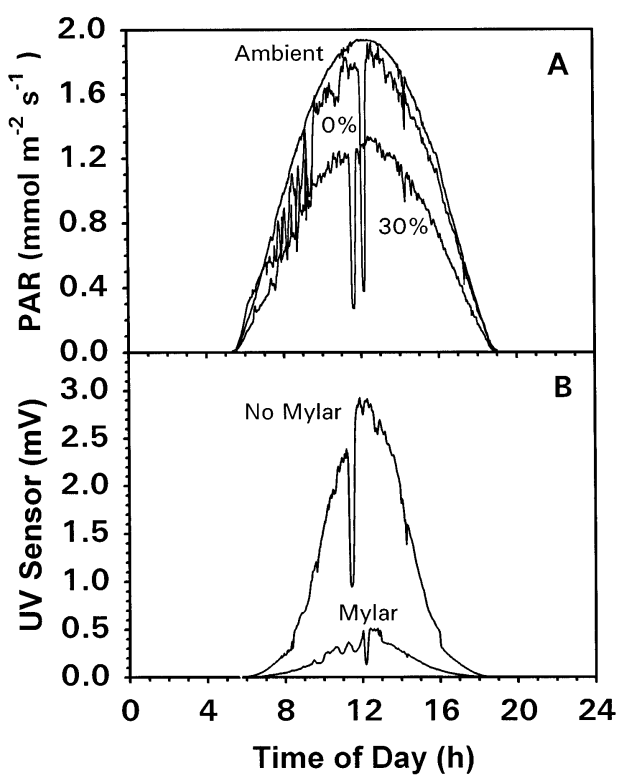

Fig. 2. Chamber daily radiation characteristics on a moderately clear day prior to the beginning of experiment 1. (A) Transmission of ambient PAR to the chamber interior without $(0 \%)$ and with $(30 \%)$ shade cloth. The ambient integral was $57.64 \mathrm{~mol} \mathrm{~m}^{-2}$ day $^{-1}$, the $0 \%$ shade treatment was 50.72 and the $30 \%$ shade treatment $36.31 \mathrm{~mol} \mathrm{~m}^{-2}$ day $^{-1}$. (B) UV transmission in the chambers without shade cloth and with and without Mylar. Ambient daily integrals were $7.23 \mathrm{~kJ} \mathrm{~m}^{-2}$ day $^{-1}$ (QDNA) and $4.82 \mathrm{~kJ} \mathrm{~m}^{-2}$ day $^{-1}$ (PAS300). Inside the chamber, the integrals were 6.44 (QDNA) and 4.29 (PAS300) $\mathrm{kJ} \mathrm{m}^{-2}$ day $^{-1}$ without the Mylar filter and 1.26 and $0.84 \mathrm{~kJ}$ $\mathrm{m}^{-2}$ day ${ }^{-1}$ with the Mylar filter installed.

mained stable even at ambient temperatures as high as $39^{\circ} \mathrm{C}$.

\subsection{Plant growth}

\subsubsection{Field filters}

Plant growth parameters for Ler and tt-5 are shown in Table 3. Clearly the growth of both Ler and $\mathrm{tt}-5$ were retarded by the low light levels during the period $1,70 \%$ shade treatment as indicated by their small diameter at 40 DAP as compared to later experiments. Ler, which showed no statistically significant differences in rosette diameter due to filter treatments throughout the experiment, was always significantly larger than tt-5. Significant UV-B effects became apparent in $\mathrm{tt}-5$ diameter growth within 5 days after the start of UV-B exposures (data not shown) and persisted until harvest. Final rosette diameter and rosette fresh weights also are shown in Table 3. Ler showed no significant differences in either parameter while the $\mathrm{CD}$ treatment retarded diameter growth in the tt -5 mutant by $24 \%$ and rosette fresh weight by $40 \%$. Probably because of the short/shortening days, these plants were entirely vegetative when harvested. In addition, $\mathrm{tt}-5$ under the $C D$ filters also exhibited cupping of existing leaves and severe distortion of new leaves, which is typical of UV stress effects reported earlier (Li et al., 1993).

It should be noted that growth rates during this experiment were very erratic owing to the lack of temperature control. There were two periods associated with mean daily temperatures of less than $5^{\circ} \mathrm{C}$ when the growth rates declined dramatically.

\subsubsection{Chamber experiment 1}

Mean final rosette diameters are shown in Table 4 . In the $30 \%$ shade treatment there were no significant UV-B effects in either genotype. However, the growth of tt-5 was significantly reduced by an average $33 \%$ compared to Ler in both filter treatments.

There was no detectable UV-B response in tt-5 under the $0 \%$ shade treatment, but there was an

Table 3

Mean plant data for field-filter exposures ${ }^{a}$

\begin{tabular}{|c|c|c|c|}
\hline Line & Mylar & $\mathrm{CD}$ & $P$ \\
\hline \multicolumn{4}{|c|}{ Rosette diameter (mm) } \\
\hline \multicolumn{4}{|c|}{ Start $(40 D A P)$} \\
\hline Ler & $26.0 \mathrm{~A}(7)$ & 26.3 A (7) & 0.828 \\
\hline tt-5 & 16.7 B (9) & $17.1 \mathrm{~B}(8)$ & 0.637 \\
\hline \multicolumn{4}{|c|}{ Final $(95 D A P)$} \\
\hline Ler & 56.5 A (7) & $53.0 \mathrm{~A}(7)$ & 0.158 \\
\hline tt-5 & 33.6 B (9) & $25.4 \mathrm{~B}(8)$ & 0.003 \\
\hline \multicolumn{4}{|c|}{ Rosette fresh weight (g) } \\
\hline Ler & 0.486 A (7) & $0.493 \mathrm{~A}(7)$ & 0.904 \\
\hline tt-5 & 0.238 B (9) & $0.144 \mathrm{~B}(8)$ & 0.005 \\
\hline
\end{tabular}

\footnotetext{
a Diameter data is given for the start of the differential filter treatment as well as for the final harvest. Population numbers $(n)$ are given in parentheses following the means. Values in the $P$ column apply to that row and numbers in a column within a parameter followed by the same letter indicate the numbers are not different at $P \leq 0.05$.
} 
Table 4

Mean rosette diameters for three mini-greenhouse experiments $^{\mathrm{a}}$

\begin{tabular}{lll}
\hline Experiment & $\begin{array}{l}\text { Diameter } \\
(\mathrm{mm})\end{array}$ & Diameter $(\mathrm{mm}) \quad P$
\end{tabular}

\begin{tabular}{|c|c|c|c|}
\hline $\begin{array}{l}\text { Experiment } 1 \\
0 \% \text { Shade }\end{array}$ & Mylar & No Filter & \\
\hline Ler & $\begin{array}{l}36.0 \mathrm{~A}, \mathrm{a} \\
(16)\end{array}$ & 49.6 A, a (15) & 0.049 \\
\hline tt-5 & $\begin{array}{l}36.8 \mathrm{~A}, \mathrm{a} \\
(13)\end{array}$ & $31.1 \mathrm{~B}, \mathrm{a}(15)$ & 0.732 \\
\hline \multicolumn{4}{|l|}{$30 \%$ Shade } \\
\hline Ler & $\begin{array}{l}68.7 \mathrm{~A}, \mathrm{~b} \\
(14)\end{array}$ & $63.4 \mathrm{~A}, \mathrm{~b}(13)$ & 0.781 \\
\hline tt-5 & $42.2 \mathrm{~B}, \mathrm{a}(15)$ & $46.1 \mathrm{~B}, \mathrm{~b}(16)$ & 0.884 \\
\hline $\begin{array}{l}\text { Experiment } 2 \\
30 \% \text { Shade }\end{array}$ & Mylar & $\mathrm{CD}$ & \\
\hline Ler & 82.6 A (17) & 73.2 A (16) & 0.083 \\
\hline tt-5 & 60.6 B (16) & 58.1 B (11) & 0.672 \\
\hline $\begin{array}{l}\text { Experiment } 3 \\
30 \% \text { Shade }\end{array}$ & PVC $\rightarrow$ Mylar & Mylar $\rightarrow$ PVC & \\
\hline \multicolumn{4}{|l|}{$\begin{array}{l}\text { Pre-swap } \\
\text { (DAP40) }\end{array}$} \\
\hline Ler & 77.0 A (17) & 81.1 A (19) & 0.188 \\
\hline tt-5 & 43.5 B (19) & 38.6 B (17) & 0.109 \\
\hline \multicolumn{4}{|l|}{ Final (DAP65) } \\
\hline Ler & 140.1 A (17) & 156.2 A (19) & $<0.001$ \\
\hline tt-5 & 70.5 B (19) & 87.1 B (17) & $<0.001$ \\
\hline
\end{tabular}

${ }^{a}$ In experiment 1 the Mylar filters were removed from half the plants in each chamber about half way through the growth period. In experiment 2 the Mylar filter on one chamber was replaced by $\mathrm{CD}$ about half way through the experiment and in experiment 3 the plants were swapped between chambers equipped with PVC and Mylar filters at DAP 40. The number of observations is given in parentheses following each mean. Values in the $P$ column apply to that particular row (filter treatment). Within experiment 1 , the lower case letters following each mean apply between shade treatments within a genotype with different letters indicating significant differences for $P<0.05$. In all experiments different capital letters following the means indicate differences $(P<0.05)$ between genotypes within a shade and filter treatment.

increase in rosette diameter of Ler under the high UV-B treatment. Comparison of genotypes and filter treatments between shade treatments reveals significantly larger plants in the $30 \%$ shade treatment, with the exception of the tt-5-Mylar treatment. The $0 \%$ Mylar treatment was the only case where there was no difference between Ler and tt-5. This lack of difference, however, was due to reduced growth of Ler and not to enhanced growth of tt-5. The generally pale aspect of all the plants in the $0 \%$ shade plot was suggestive of photo bleaching, characteristic of very high light damage. In addition, the greater growth of Ler in the high UV-B plot, from which the Mylar shields had been removed, suggests that another stressor, perhaps high temperatures may have been occurring under the Mylar shields. In any case, it is clear that Ler was being damaged in this plot and did not constitute an adequate reference for comparing physiological responses under such conditions. Therefore, the $0 \%$ shade plot will be eliminated from further consideration and all further references to chamber experiment 1 will be confined to the $30 \%$ shade plot.

The harvest fresh weight data for the $30 \%$ shade plot are given in Table 5. None of the direct measures showed any significant UV-B effects in either genotype while the data for $\mathrm{tt}-5$ are generally of the order of $50 \%$ of that for Ler. The only significant UV-B effect was in the harvest index (HI) (reproductive \%) of tt-5 which showed an increase in the high UV-B plot.

Measurements of total phenolics and $a b-$ sorbance at $300 \mathrm{~nm}$ (Table 6) Showed the expected responses to increased UV-B exposure. The Ler genotype showed significant increases in both total phenolics $(21 \%)$ and $A_{300}(24 \%)$ as would normally happen in a fully functional system. In contrast, the $\mathrm{tt}-5$ genotype showed no significant change in either parameter in response to increased UV-B, but did show 39\% less total phenolics and $44 \%$ less $A_{300}$ on average when compared to Ler. The lack of a significant difference in total phenolics in tt-5, which might appear marginally significant in Table 5, was confirmed in subsequent experiments (unpublished data).

\subsubsection{Chamber experiment 2}

Again, there are no significant UV-B effects on the mean final rosette diameter in either genotype (Table 4) and the tt-5 rosettes are smaller than Ler under both Mylar and CD by an average of $24 \%$. Neither the total dry weight, total fresh weight nor the total aboveground vegetative fresh weight showed any significant UV-B effects (Table 5). There was, however, a significant (29\%) 
increase in reproductive fresh weight in the $\mathrm{tt}-5$ genotype leading to a significant increase in the HI in the high UV-B plot. Interestingly, in Ler a non-significant increase in reproductive fresh weight combined with a non-significant decrease

Table 5

Arabidopsis harvest parameters for chamber experiments 1 and $2^{\mathrm{a}}$

\begin{tabular}{|c|c|c|c|}
\hline Experiment 1 & Mylar & No filter & $P$ \\
\hline \multicolumn{4}{|c|}{ Total fresh weight $(g)$} \\
\hline Ler & $5.06 \mathrm{~A}(14)$ & $4.91 \mathrm{~A}(13)$ & 0.440 \\
\hline $\mathrm{tt}-5$ & $2.51 \mathrm{~B}(15)$ & $2.32 \mathrm{~B}(16)$ & 0.380 \\
\hline \multicolumn{4}{|c|}{ Rosette fresh weight $(g)$} \\
\hline Ler & $0.458 \mathrm{~A}$ & $0.494 \mathrm{~A}$ & 0.747 \\
\hline tt-5 & $0.226 \mathrm{~B}$ & $0.244 \mathrm{~B}$ & 0.762 \\
\hline \multicolumn{4}{|c|}{ Stem fresh weight $(g)$} \\
\hline Ler & $2.095 \mathrm{~A}$ & $1.882 \mathrm{~A}$ & 0.495 \\
\hline $\mathrm{tt}-5$ & $0.880 \mathrm{~B}$ & $0.717 \mathrm{~B}$ & 0.093 \\
\hline \multicolumn{4}{|c|}{ Leaf fresh weight $(g)$} \\
\hline Ler & $0.845 \mathrm{~A}$ & $0.791 \mathrm{~A}$ & 0.719 \\
\hline tt-5 & $0.486 \mathrm{~B}$ & $0.397 \mathrm{~B}$ & 0.101 \\
\hline \multicolumn{4}{|c|}{ Reproductive fresh weight ( $g$ ) } \\
\hline Ler & $1.849 \mathrm{~A}$ & $1.740 \mathrm{~A}$ & 0.490 \\
\hline $\mathrm{tt}-5$ & $0.922 \mathrm{~B}$ & $0.958 \mathrm{~B}$ & 0.440 \\
\hline \multicolumn{4}{|c|}{ Reproductive (\%) } \\
\hline Ler & $34.9 \mathrm{~A}$ & $35.1 \mathrm{~A}$ & 0.916 \\
\hline tt-5 & $37.2 \mathrm{~A}$ & $43.8 \mathrm{~B}$ & 0.003 \\
\hline Experiment 2 & Mylar & $\mathrm{CD}$ & $P$ \\
\hline \multicolumn{4}{|c|}{ Total dry weight $(g)$} \\
\hline Ler & 0.990 A (17) & $1.020 \mathrm{~A} \mathrm{(16)}$ & 0.549 \\
\hline $\mathrm{tt}-5$ & $0.500 \mathrm{~B}(16)$ & $0.433 \mathrm{~B}(11)$ & 0.318 \\
\hline \multicolumn{4}{|c|}{ Total fresh weight $(\mathrm{g})$} \\
\hline Ler & $8.79 \mathrm{~A}$ & $7.95 \mathrm{~A}$ & 0.435 \\
\hline $\mathrm{tt}-5$ & $4.50 \mathrm{~B}$ & $4.61 \mathrm{~B}$ & 0.784 \\
\hline \multicolumn{4}{|c|}{ Other fresh weight $(g)$} \\
\hline Ler & $6.01 \mathrm{~A}$ & $5.03 \mathrm{~A}$ & 0.186 \\
\hline $\mathrm{tt}-5$ & $3.35 \mathrm{~B}$ & $3.13 \mathrm{~B}$ & 0.467 \\
\hline \multicolumn{4}{|c|}{ Reproductive fresh weight $(g)$} \\
\hline Ler & $2.77 \mathrm{~A}$ & $2.92 \mathrm{~A}$ & 0.708 \\
\hline $\mathrm{tt}-5$ & $1.15 \mathrm{~B}$ & $1.48 \mathrm{~B}$ & 0.0316 \\
\hline \multicolumn{4}{|c|}{ Reproductive (\%) } \\
\hline Ler & $31.1 \mathrm{~A}$ & $37.3 \mathrm{~A}$ & 0.0106 \\
\hline $\mathrm{tt}-5$ & $25.4 \mathrm{~B}$ & $31.9 \mathrm{~B}$ & 0.0017 \\
\hline \multicolumn{4}{|l|}{ Height (cm) } \\
\hline Ler & $26.9 \mathrm{~A}$ & $25.4 \mathrm{~A}$ & 0.136 \\
\hline tt-5 & $20.7 \mathrm{~B}$ & $6.8 \mathrm{~B}$ & 0.0005 \\
\hline
\end{tabular}

a Leaf FW is for leaves other than in the rosette. The number of observations is indicated in parentheses following the means for total fresh weight in experiment 1 and total dry weight in experiment 2. $P$ values apply to that particular row and numbers in a column within a parameter followed by the same letter indicate the numbers are not different at $P \leq 0.05$. in total fresh weight to produce a significant increase in HI. Harvest plant height measurements showed a $19 \%$ decrease in tt-5. All plant parameters measured in this experiment showed significantly lower values for the tt-5 mutant when compared to Ler.

\subsubsection{Chamber experiment 3}

Mean rosette diameters were the only plant measurements made during this experiment and prior to swapping the plants between chambers on DAP 40, neither genotype showed any UV-B effects, although the $\mathrm{tt}-5$ genotype averaged $48 \%$ smaller than Ler (Table 4). By contrast, the final diameters for both genotypes showed significant UV-B effects. The Ler plants that spent their last 25 days of growth under the PVC filters averaged $12 \%$ larger than those that finished under the Mylar. In the case of $\mathrm{tt}-5$, that same comparison showed a $24 \%$ difference. The tt-5 genotype showed a $47 \%$ average reduction in growth compared to Ler under both filter treatments.

\section{Discussion}

\section{1. $U V-B$ responses}

In the experiments reported here, detection of significant UV-B responses within the $\mathrm{tt}-5$ genotype has been problematical. Rosette diameter growth appears to work well as long as the plants remain vegetative (field filter experiment) and damage is given sufficient time to accumulate. However, once reproductive growth is initiated resources are reallocated toward seed production, then rosette growth slows to a halt followed by senescence. This sequence of events leaves little opportunity to detect a UV-B response unless the UV-B treatments are applied earlier during the vegetative phase than was done in chamber experiments 1 and 2. In those cases the rosettes had already reached 84 and $80 \%$, respectively, of their maximum size. However, in the field filter experiment and chamber experiment 3, UV-B manipulations were conducted when the rosettes had reached only about $50 \%$ of their eventual diameter, thus leaving adequate time for damage to 
Table 6

Total phenolics and UV-absorbing compounds $\left(A_{300}\right)$ for experiment $1(30 \% \text { shade in Table } 3)^{\mathrm{a}}$

\begin{tabular}{|c|c|c|c|c|}
\hline & Line & Mylar & Filter removed & $P$ \\
\hline Total phenolics (mg) & Ler & 19.88 A (5) & $24.10 \mathrm{~A}(5)$ & 0.038 \\
\hline \multirow[t]{2}{*}{ Absorbance $_{300}$} & Ler & $0.943 \mathrm{~A}(5)$ & $1.168 \mathrm{~A} \mathrm{(5)}$ & 0.030 \\
\hline & $\mathrm{tt}-5$ & $0.554 \mathrm{~B}(5)$ & $0.626 \mathrm{~B}(2)$ & 0.570 \\
\hline
\end{tabular}

\footnotetext{
a Phenolics are given as 4-coumaric acid equivalents $(\mathrm{g} \text { dry wt })^{-1} . P$ values are given for comparisons within a line while different letters following the means for each line indicate differences between lines significant at $P \leq 0.05$. Number of samples is in parentheses following each mean.
}

accumulate and be expressed. Rosette fresh weights, although not measured in chamber experiments 2 and 3, showed significant UV-B effects in the field filter experiment while no difference was detected in chamber experiment 1 . This discrepancy, like the diameter measurements, was probably because in the former experiment, plants were still vegetative when harvested and in the latter, they were reproductive and the rosettes were already senescing.

There were no significant UV-B effects in any of the fresh weight categories of experiment 1 , even in the tt-5 genotype. However, even though the fresh weight effects were not significant nor especially pronounced, examination of the reproductive fresh weight as a fraction of the total shows a typical and significant stress response in $\mathrm{tt}-5$. The so-called reproductive effort, which is very similar to the economic HI for crop plants, showed an $18 \%$ increase even while the total fresh weight exhibited a slight (non-significant) decline. There was no change in the HI of Ler. The change in $\mathrm{HI}$ of $\mathrm{tt}-5$ was confirmed in chamber experiment 2 where the cause could be traced to a significant increase in reproductive fresh weight. However, Ler also showed an increase in HI, suggesting that it too was subject to some radiation stress. Comparison of HI between experiments 1 and 2 suggests the possibility that both tt-5 and Ler could have been somewhat stressed even under the Mylar filters in experiment 1 since $\mathrm{HI}$ values were higher in both genotypes than in chamber experiment 2. Although ambient UV-B and PAR radiation levels were about 20\% lower in period 2 of experiment 2, the proportional exclusion of UV-B was also much better than in experiment 1; compare the $90 \%+$ exclusion of the Mylar filters in experiment 2 with the $80 \%+$ exclusion during experiment 1 . Probably because of a high proportion of diffuse radiation in the UV$\mathrm{B}$ band and owing to reflections within the chambers, it was very difficult to achieve maximum UV-B exclusion by segregating populations within a single chamber. In any case, the plants under the Mylar shields in experiment 1 were exposed to about three times the UV-B radiation as those in experiment 2. Thus, it is possible that the HI of both lines was already high under the Mylar in experiment 1 , whereas better UV-B exclusion under the Mylar in experiment 2 may have lowered $\mathrm{HI}$ and allowed a greater range of expression in both genotypes. Also, there is the possibility, which cannot be discounted, that the Mylar shields in experiment 1 interfered with air flow sufficiently to cause unmonitored heating of the plants and rooting medium. This effect would be especially evident in the unshaded chamber where all the plants clearly experienced stresses great enough to significantly retard growth. Resolution of these questions will require further experimentation. Nonetheless, it appears that HI may be a fairly sensitive and reliable stress indicator in this system but, unfortunately is very tedious to measure if many plants are involved.

Plant height at harvest, only measured in experiment 2 , showed a very clear UV-B effect in tt-5 with a $19 \%$ inhibition in height growth over the 11 days of the UV-B exposure. This is an easily measured parameter and may provide a sensitive indicator of stress in reproductive Arabidopsis plants. 
The fact that tt-5 mutants are deficient in certain phenolic and UV-B absorbing compounds was confirmed by measurement of these metabolites in soluble extracts (Table 6). In Ler, both the total phenolics and $A_{300}$ were at relatively high levels even under the Mylar shields, probably due to the relatively high levels of visible radiation and the UV-A that accompanies it which have been shown, in addition to UV-B, to stimulate production of UV-absorbing compounds (Warner and Caldwell, 1983; Beggs et al., 1986; Cen and Bornman, 1990). Nonetheless, both of these factors showed significant increases in Ler as a result of removing the Mylar UV-B shields (Table 6), stimulated either by the $17 \%$ increase in PAR and UV-A or by the 4-fold increase in UV-B (Table 2). In either case the stimulation appears to be additive in nature and indicates that the UV-B levels and differential filter treatments were adequate to stimulate a normal plant response despite the general absence of a manifestation of that response in terms of harvest biomass parameters. On the other hand, tt-5 showed no significant change in either total phenolics or $A_{300}$ (Table 6).

\subsection{Genotype differences}

With the exception of the comparison of $\mathrm{HI}$ under the Mylar shields in the 30\% shade plot of experiment 1 and the anomalous behavior of Ler in the $0 \%$ shade treatment of that experiment, rosette diameters and all harvest data cleanly segregate $(P \leq 0.05)$ between Ler and tt-5 under all filter treatments in all experiments. Averaged across all experiments, growth suppression of $\mathrm{tt}-5$ relative to Ler was $39 \%$ for rosette diameter and $51 \%$ for all the harvest parameters combined. These observations suggest either: (1) an extreme sensitivity to very low levels of UV-B (especially experiments 2 and 3); (2) that the damaging radiation band extends well into the UV-A region, not excluded by the Mylar, as shown by Quaite and Sutherland (1992) for DNA damage in alfalfa; (3) that metabolites or processes downstream from chalcone isomerase are critical for normal growth even in the absence of UV-B radiation; or (4) that other genes were affected along with chalcone isomerase during development of the tt-5 mutant.
Although the tt-5 mutant involves an inversion and deletion that affects sites at other locations, analysis suggests that the tt-5 lesion is essentially localized within the chalcone isomerase gene and that no additional genes are disrupted (Shirley et al., 1992). Therefore, we feel that option number (4) is unlikely to explain the growth difference between $\mathrm{tt}-5$ and Ler. Option (1) is an unlikely candidate since Li et al. (1993) showed growth suppression in tt-5 relative to Ler in the absence of UV-B and we observed a 44\% suppression of tt-5 grown under PVC, which excludes virtually all PAS300 and QDNA radiation. In the case of $\mathrm{Li}$ et al. (1993), the reduction was about $18 \%$ averaged over their three experiments, compared to an average, over all experiments and measured parameters, of $45 \%$ in ours. The larger reduction in our experiments may have been due to less than complete UV-B exclusion or again, to the higher levels of UV-A that occurred even under the Mylar filters. That the latter (option (2)) is unlikely to provide the explanation is supported by experiment 3 in which the Ler/tt-5 growth differential (44\%) was clearly established under both Mylar and PVC filters prior to swapping the plants between chambers. Although there appeared to be some additional effect of the higher UV-A exposure under the Mylar, a better determination of that effect will require a more refined experiment. However, it is clear from this experiment that even excluding UV-A out to $340 \mathrm{~nm}$ is not sufficient to overcome the growth inhibiting effects of the tt-5 mutation. All of the experiments reported here, taken together with those of $\mathrm{Li}$ et al. (1993) strongly support the notion that other metabolites or processes downstream from chalcone isomerase may be critical for normal growth even in the absence of damaging UV-B radiation (option (3)). Furthermore, even though there is a positive correlation between the intensity of solar radiation and the quantity of phenolics produced by plants, there might be factors regulating production of phenolics that are only partly associated with UV protection (Lovelock et al., 1992). Indeed, it has been argued that production of carbon-based secondary products serves to balance the supply of photosynthate with the availability of other resources or environmental 
conditions required for growth (Waterman and Mole, 1994, pp. 57-66). Flavonoids and their metabolism are involved in numerous plant-environment interactions (Waterman and Mole, 1994, pp. 57-66), which might be indicative of their importance in normal growth and differentiation processes occurring within the constraints of various environmental factors. It thus seems plausible to us that the failure of the tt-5 mutants to grow normally, even at very low UV-B levels, is not due merely to its inability to synthesize flavonoids to screen out UV-B, but is related to other imbalances between normal growth and differentiation processes involving secondary metabolism downstream from the chalcone isomerase site. We must then reject our original hypothesis that given radiation with an otherwise normal spectral distribution but lacking UV-B, tt-5 will grow as well as Ler and conclude that tt-5 is unsuitable for studies of normal UV-B response mechanisms.

\section{Conclusions}

The Teflon-covered chambers appeared to work very much as anticipated providing satisfactory transmission of natural UV and visible radiation and temperature control adequate for the growth of Arabidopsis.

While rosette diameter may be a reasonable non-destructive measure of plant growth and environmental treatment effects prior to bolting, there is only a short time after bolting during which realistic environmental effects can be detected by this means. Harvest index appears to be a sensitive indicator of radiation stress in reproductive Arabidopsis but is time-consuming to measure on a routine basis. However, plant height may provide an easily measured non-destructive indicator of UV-B response in these circumstances. Finally, the substantial suppression of growth of $\mathrm{tt}-5$ compared to Ler in all the experiments reported here and especially under Mylar and PVC filters suggests that the disruption of secondary metabolism at the chalcone isomerase site is affecting some other, perhaps more than one, critical aspect of secondary metabolism. This critical aspect, which seems to be independent of the plants ability to screen out UV-B, results in an impairment of the normal growth and differentiation processes thus, severely limiting its usefulness as a surrogate for mechanistic high UV-B studies.

\section{References}

Beggs, C.J., Schneider-Ziebert, U., Wellmann, E., 1986. UV-B radiation and adaptive mechanisms in plants. In: Worrest, R.C., Caldwell, M.M. (Eds.), UV-B Radiation and Adaptive Mechanisms in Plants. Springer, Berlin, pp. 235-250.

Björn, L.O., Murphy, T.M., 1985. Computer calculation of solar ultraviolet radiation at ground level. Physiol. Veg. 23, $555-561$.

Caldwell, M.M. 1971. Solar ultraviolet radiation and the growth and development of higher plants. In: Giese, A.C. (Ed.). Solar ultraviolet radiation and the growth and development of higher plants. Academic Press, New York, pp. $131-177$.

Caldwell, M.M., Flint, S.D., Searles, P.S., 1994. Spectral balance and UV-B sensitivity of soybean: a field experiment. Plant Cell Environ. 17, 267-276.

Cen, Y., Bornman, J.F., 1990. The response of bean plants to UV-B radiation under different irradiances of background visible light. J. Exp. Bot. 41, 1489-1495.

Fiscus, E.L., Booker, F.L., 1994. UV-B: An adaptation of Green/Björn and Murphy (A computer program available from first author on request). US Department of Agriculture, Raleigh, NC.

Fiscus, E.L., Booker, F.L., 1995. Is increased UV-B a threat to crop photosynthesis and productivity? Photosynth. Res. 43, 81-92.

Green, A.E.S., 1983. Penetration of ultraviolet radiation to the ground. Physiol. Plant. 58, 351-359.

Koornneef, M., 1981. The complex syndrome of TTG mutants. Arab. Inform. Serv. 18, 45-51.

Landry, L.G., Chapple, C.C.S., Last, R.L., 1995. Arabidopsis mutants lacking phenolic sunscreens exhibit enhanced ultraviolet-B injury and oxidative damage. Plant Physiol. 109, 1159-1166.

Li, J., Ou-Lee, T.-M., Raba, R., Amundson, R.G., Last, R.L., 1993. Arabidopsis flavonoid mutants are hypersensitive to UV-B radiation. Plant Cell 5, 171-179.

Lovelock, C.E., Clough, B.F., Woodrow, I.E., 1992. Distribution and accumulation of ultraviolet-radiation-absorbing compounds in leaves of topical mangroves. Planta 188, $143-154$.

Quaite, F.E., Sutherland, B.M., Sutherland, J.C., 1992. Action spectrum for DNA damage in alfalfa lowers predicted impact of ozone depletion. Nature 358, 576-578.

Robberecht, R., Caldwell, M.M., 1978. Leaf epidermal transmittance of ultraviolet radiation and its implications for plant sensitivity to ultraviolet-radiation induced injury. Oecologia 32, 277-287. 
Ryan, K.G., Ireland, W., 1997. A small-scale outdoor growth chamber with modulated enhancement of solar UV-B radiation. J. Environ. Qual. 26, 866-871.

Shirley, B.W., Hanley, S., Goodman, H.M., 1992. Effects of ionizing radiation on a plant genome: analysis of two Arabidopsis transparent testa mutation. Plant Cell 4, $333-$ 347.

Singleton, V.L., Rossi, J.A., 1965. Colorimetry of total pheno- lics with phosphomolybdic phosphotungstic acid reagents. J. Enol. Viticult. 16, 144-158.

Warner, C.W., Caldwell, M.M., 1983. Influence of photon flux density in the $400-700 \mathrm{~nm}$ waveband on inhibition of photosynthesis by UV-B $(280-320 \mathrm{~nm})$ irradiation in soybean leaves. Photochem. Photobiol. 38, 341-346.

Waterman, P.G., Mole, S., 1994. Analysis of Phenolic Plant Metabolites. Blackwell, London 238 pp. 